\title{
Diverse Effects of Small Molecule Inhibitors on Actin Cytoskeleton Dynamics in HIV-1 Infection
}

\author{
YoungHyun Shin, Byeong-Sun Choi, Kyung-Chang Kim, Kisoon Kim and Cheol-Hee Yoon ${ }^{*}$ \\ Division of Viral Disease Research, Center for Infectious Disease Research, Korea National Institute of Health, \\ Chungbuk, Republic of Korea
}

\author{
Corresponding \\ Cheol-Hee Yoon Ph.D. \\ Division of Viral Disease Research, Center for \\ Infectious Disease Research, Korea National \\ Institute of Health, 187 Osongsaengmyeong \\ 2-ro, Osong-yeup, Cheongju, Chungbuk, \\ 363-951, South Korea \\ Phone : +82-43-719-8414 \\ Fax : +82-43-719-8459 \\ E-mail : kmc755@korea.kr
}

Received : February 15, 2019

Revised : April 4, 2019

Accepted : April 5, 2019
No potential conflict of interest relevant to this article was reported.

Copyright (C) 2019 Journal of Bacteriology and Virology

(OThis is an Open Access article distributed under the terms of the Creative Commons Attribution Non-Commercial License (http://creativecommons.org/ license/by-nc/3.0/).
The dynamics of the actin cytoskeleton plays a pivotal role in the process of cell division, the transportation of organelles, vesicle trafficking and cell movement. Human immunodeficiency virus type 1 (HIV-1) hijacks the actin dynamics network during the viral entry and migration of the pre-integration complex (PIC) into the nucleus. Actin dynamics linked to HIV-1 has emerged as a potent therapeutic target against HIV infection. Although some inhibitors have been intensely analyzed with regard to HIV-1 infection, their effects are sometimes disputed and the exact mechanisms for actin dynamics in HIV infection have not been well elucidated. In this study, the small molecules regulating HIV-1 infection from diverse inhibitors of the actin dynamic network were screened. Two compounds, including Chaetoglobosin A and CK-548, were observed to specifically bar the viral infection, while the cytochalasin family, 187-1, N-WASP inhibitor, Rho GTPase family inhibitors (EHop-016, CID44216842, and ML-141) and LIMK inhibitor (LIM domain kinase inhibitor) increased the viral infection without cytotoxicity within a range of $\sim \mu \mathrm{M}$. However, previously known inhibitory compounds of HIV-1 infection, such as Latrunculin A, Jasplakinolide, Wiskostatin and Swinholide A, exhibited either an inhibitory effect on HIV-1 infection combined with severe cytotoxicity or showed no effects. Our data indicate that Chaetoglobosin A and CK-548 have considerable potential for development as new therapeutic drugs for the treatment of HIV infection. In addition, the newly identified roles of Cytochalasins and some inhibitors of Rho GTPase and LIMK may provide fundamental knowledge for understanding the complicated actin dynamic pathway when infected by HIV-1. Remarkably, the newly defined action modes of the inhibitors may be helpful in developing potent anti-HIV drugs that target the actin network, which are required for HIV infection.

Key Words: Actin dynamics network, Small molecule inhibitors, HIV infection, anti-HIV effect

\section{INTRODUCTION}

Human immunodeficiency virus type 1 (HIV-1) is a causative agent for acquired immunodeficiency syndrome (AIDS), which is a global public health hazard (1). Currently, six classes of anti-retroviral drugs targeting viral proteins have been developed to treat AIDS patients, and which are proven to effectively inhibit viral replication and extend a patient's life span. However, the treatment does not eliminate completely the virus infection and, consequently, drug-resistant mutations pools are expanding due to sustained long-term treatment with antiviral drugs (2). The emergence of drug resistance inducing viral proteins has 
alternatively led to an effort to develop targeting host key factor interacting with HIV during viral infection.

The dynamic remolding of the actin cytoskeleton is a critical part of most cellular activities, and the cooperative action of cytoskeletal proteins results in various cellular responses, including directional cell movement, maintenance of the cell shape, intracellular organelle migration, and vesicle trafficking, etc. The harmonized regulation of the assembly and disassembly of the actin filaments is achieved through signaling cascade by multiple proteins $(3,4)$.

The molecular network was targeted by infectious microorganisms to induce their infectivity, which resulted in pathogenesis. The vaccinia virus encodes the A36R protein, which recruits the host N-Wiskott-Aldrich syndrome protein (N-WASP) to induce Arp2/3-mediated filament nucleation, and then promotes the intracellular migration of the enveloped virus (5). The intracellular migration of bacculovirus and nuclear entry were required early on in the viral infection process, wherein p78/83 of bacculovirus directly recruited Arp2/3 complex, and then promoted actin filament polymerization (6).

With regard to HIV-1 infection, it has been reported that HIV proteins (RTase, Gag and Nef) were integrated in the regulation of the actin filament. Notably, the Nef-mediated regulation of actin dynamics was well elucidated regarding the Nef-induced inhibition of cell movement. Nef binds to the actin skeleton regulatory kinase, PAK2 (p21 activated kinase 2) and DOCK2 (dedicator of cytokinesis 2)-ELMO1 (engulfment and cell motility protein 1) complex key activator of Rac, and this binding alters cofilin-induced actin depolymerization and Rac GTPase activity, respectively (7), thereby restricting the migration of infected $\mathrm{T}$ lymphocytes. Although the Nef-mediated regulation of the actin cytoskeleton may be considered a critical mechanism based on Nef-induced virulence after infection, the dynamic regulation of the cortical actin skeleton linked to HIV-1 infection has not been well elucidated because the large quantity of data involved was too scattered and complicated to interpret the exact pathway.

The generally accepted actin dynamic mechanism in HIV infection includes the initial signal started by cell surface receptors (CD4/CXCR4 or CCR5) after viral gp120 attachment. The signals are transmitted to the Rho family GTPase (Rho, Rac and Cdc42), and the signaling is then dispatched to actin skeleton effector proteins such as PAK1/2, LIMK1, WASPS (Wiskott-Aldrich syndrome proteins), WAVE2 (WASP-family verprolin-homologous protein 2), Arp2/3, profilin, cofilin, and actin, etc., which follows facilitation of viral membrane fusion to the host membrane, a de-coating process, and the migration of the PIC. However, a large portion of the mechanism by which HIV utilizes the actin cytoskeleton to infect the host remains to be elucidated $(8,9)$.

Numerous small molecules have been discovered that mechanistically mimic the host regulatory mechanism for modulating the actin cytoskeleton, such as crosslinking, capping, sequestering, and serving. In addition, some compounds have been observed to inhibit the Arp2/3 both directly and indirectly by inhibiting nucleation promoting factors (NPFs) such as WASP and WAVEs. A limited number of compounds, including latrunculins, cytochalasins, jasplakinolide, and wiskostain, have been studied in order to understand the viral infection mechanism utilizing the actin cytoskeleton with regard to HIV infection; and several actin dynamic mechanisms discovered by these compounds have been suggested as potent therapeutic targets for HIV treatment (10-13). Nevertheless, their inhibitory effects were controversial according to experimental conditions and cell types, making it complicated to interpret the interaction between actin networks and HIV-1. Therefore, a screening of the regulatory compounds of actin dynamics with regard to HIV-1 infection was considered by this study to define the precise mechanism underlying the utilization of actin dynamics for viral infection and to devise a novel strategy for developing therapeutic agents against HIV infection based on regulation of the actin dynamic network.

This study used screening compounds with an HIV-1 regulatory effect from among commercially available inhibitors of actin cytoskeleton dynamics. Of these, three compounds showed an inhibitory effect on HIV-1 infection while, conversely, eleven compounds exhibited increased HIV-1 infectivity at concentrations showing low cell toxicity. These regulatory effects were further confirmed with a dose-response assay. Although some of these had been studied previously in an effort to understand the viral utilization of actin networks during HIV-1 infection, inhibitors such as cytochalasins showed different 
regulatory patterns on viral infection compared with previous reports. Additionally, our study defined novel roles for several inhibitors of HIV infection and production.

\section{MATERIALS AND METHODS}

\section{Cell culture, virus and reagents}

For the purposes of this study, TZM-bl cells, ACH2 and the HIV-1 clone pNL4-3 were obtained from the NIH AIDS Research and Reference Reagent Program (NIH/NIAID). The cells were cultured as previously described (14). Briefly, the cells were cultured in the RPMI medium (Gibco-BRL, Waltham, MA, USA) or in Dulbecco's modified Eagle's medium (DMEM, Gibco-BRL) supplemented with $1 \%$ penicillin-streptomycin and $10 \%(\mathrm{v} / \mathrm{v})$ heat-inactivated fetal bovine serum (FBS, Gibco-BRL). The preparation of infectious HIV-1 was performed as described previously (14). Briefly, $15 \mu \mathrm{g}$ of molecular HIV-1 clones (HIV-1 clone pNL4-3) was transfected into 293T cells using Lipofectamine 2000 (Invitrogen, Carlsbad, CA, USA). At $48 \mathrm{~h}$ after transfection, the supernatants were harvested and filtered to remove any cell debris. The titers of the HIV-1 particles in the supernatant were assessed using an HIV-1 p24 ELISA kit (PerkinElmer, Waltham, MA, USA), and the aliquots were stored at $-80^{\circ} \mathrm{C}$ until use. Small inhibitory molecules for the actin cytoskeletal pathway listed in Table 1 were purchased from Sigma Aldrich (St. Louis, MO, USA) and Tocris Bioscience (Bristol, UK). The mode of action of the inhibitors is described in Table 1.

\section{Primary screening of compounds with a viral-infectivity assay}

For the primary screening of the compounds, $1 \times 10^{4} \mathrm{TZM}$-bl cells were seeded in 96-well white plates. At $24 \mathrm{~h}$ after seeding, the cells were treated with each compound contained at a final concentration of $6.25 \mu \mathrm{M}$, and then infected with HIV-1 NL4-3 virus stock at a multiplicity of infection (MOI) of 0.1. At $48 \mathrm{~h}$ after infection, firefly-luciferase (F-Luc) activities indicating the amount of viral infection were assessed by using the Bright-Glo Luciferase Assay System (Promega, Madison, WI, USA) according to the manufacturer's instructions. The negative control was an appropriate volume of DMSO.

\section{Dose-response assay of compounds on viral infectivity}

A dose-response assay of compounds on viral infectivity was conducted to determine the inhibitory potency of effective compounds on viral infection, as described previously with minor modifications (15). Briefly, $1 \times 10^{4} \mathrm{TZM}$-bl cells were seeded on 96-well plates and infected with HIV-1 NL4-3 virus stock obtained from the transfection of 293T cells at a multiplicity of infection (MOI) of 0.1 for $4 \mathrm{~h}$. Triplicate wells cultured with $1 \times 10^{4} \mathrm{TZM}$-bl cells were treated with serially diluted compounds at concentrations ranging from $0.19-100 \mu \mathrm{M}$. At $48 \mathrm{~h}$ after infection, infectivity was assessed by measuring luminescence using a SpectraMax Paradigm Multimode Microplate Reader (Molecular Devices, San Jose, CA, USA) as explained in the section above. The infectivity data are presented as a relative percentage compared with the untreated control. Concurrently, the cytotoxicity of the compounds was determined as explained below. A 3-2,5-diphenyltetrazolium bromide (MTT)-based assessment of cell viability was performed using the PrestoBlue Cell Viability Reagent (Thermo Fisher Scientific, Waltham, MA, USA) according to the manufacturer's instructions. All the graphical data are expressed as relative percentages of infectivity and cell viability compared with those obtained in the absence of a compound, and the data are presented as the mean \pm standard deviation (SD) ( $n=3)$.

\section{Viral production assay}

The inhibitory effect of compounds on viral production was determined as described previously with some minor modifications (14). In brief, $2 \times 10^{4} \mathrm{ACH} 2$ and $\mathrm{J} 1.1$ cells were cultured in the presence or absence of effective compounds at a final concentration of $12.5 \mu \mathrm{M}$ in the presence of PMA $(0.5 \mu \mathrm{g} / \mathrm{mL}) /$ ionomycin $(1 \mu \mathrm{g} / \mathrm{mL})$. At $48 \mathrm{~h}$ after treatment, the 
Table 1. Comparison of viral infectivity on actin inhibitors

\begin{tabular}{|c|c|c|c|c|}
\hline \multirow{2}{*}{ Group } & \multirow{2}{*}{ Actin inhibitors } & \multirow{2}{*}{ Mode of action } & \multirow{2}{*}{$\begin{array}{l}\text { Infectivity }(6.3 \mu \mathrm{M}) \\
\text { Average } \pm \mathrm{SD}(\%)\end{array}$} & \multirow{2}{*}{$\begin{array}{c}\text { Cell viability }(6.3 \mu \mathrm{M}) \\
\text { Average } \pm \text { SD }(\%)\end{array}$} \\
\hline & & & & \\
\hline \multirow{3}{*}{ Group 1} & Chaetoglobosin A & $\begin{array}{l}\text { Targets filamentous actin and demonstrate antibacterial and nematicidal } \\
\text { effects as well as induces apoptosis in cancer cell lines }\end{array}$ & $19.6 \pm 4.1$ & $85.2 \pm 3.8$ \\
\hline & CK-548 & Inhibits the activity of actin-related protein $($ Arp $) 2 / 3$ complex & $48.3 \pm 2.8$ & $101.2 \pm 0.8$ \\
\hline & $T R 100$ & Selectively disrupts Tm5NM1/2 containing actin filaments & $25.7 \pm 14.4$ & $65.7 \pm 7.0$ \\
\hline \multirow{11}{*}{ Group /I } & 187-1, N-WASP inhibitor & $\begin{array}{l}\text { Prevents the activation of actin-related protein } 2 / 3 \text { (Arp2/3) complex by } \\
\text { inhibiting the interaction between } N \text {-WASP and Arp } 2 / 3\end{array}$ & $189.9 \pm 19.0$ & $93.5 \pm 2.1$ \\
\hline & Cytochalasin B & Inhibits the elongation and shortening of actin filaments & $175.7 \pm 8.6$ & $59.8 \pm 2.4$ \\
\hline & Cytochalasin D & Blocks actin polymerization & $223.6 \pm 26.0$ & $75.0 \pm 4.2$ \\
\hline & Cytochalasin $E$ & $\begin{array}{c}\text { Analog of cytochalasin B which potently and selectively inhibits the growth of } \\
\text { endothelial }\end{array}$ & $109.5 \pm 4.8$ & $62.2 \pm 2.9$ \\
\hline & Cytochalasin H & Potent inhibitor of actin incorporation into filaments & $176.9 \pm 30.4$ & $69.9 \pm 2.4$ \\
\hline & Dihydrocytochalasin $B$ & Inhibits actin assembly & $112.5 \pm 10.0$ & $79.0 \pm 2.8$ \\
\hline & EHop-016 & Potent and specific inhibitor of Rac1 and Rac3 GTPase activity & $237.9 \pm 8.4$ & $92.4 \pm 1.9$ \\
\hline & CID44216842 & Cdc42 Protein Inhibitor & $210.6 \pm 9.7$ & $99.4 \pm 0.1$ \\
\hline & $M L 141$ & Potent, selective inhibitor of the $R$ ho family $G T P a s e$ cdc 42 & $115.7 \pm 0.8$ & $88.9 \pm 6.5$ \\
\hline & T56-LIMKi & Selective LIM kinase 2 (LIMK2) inhibitor, inhibits cofilin phosphorylation & $110.6 \pm 5.3$ & $94.2 \pm 1.4$ \\
\hline & IMM-01 & $\begin{array}{l}\text { Agonist of the mammalian Diaphanous (mDia)-related formins that trigger } \\
\text { actin assembly and microtubule stabilization }\end{array}$ & $122.9 \pm 7.0$ & $104.0 \pm 2.5$ \\
\hline \multirow{14}{*}{ Group III } & Cytochalasin A & $\begin{array}{l}\text { Induces the phosphorylation of the tyrosine phosphatase PTP3 of } \\
\text { Dictyostelium, activating STATC }\end{array}$ & $43.0 \pm 3.5$ & $43.3 \pm 0.7$ \\
\hline & CK 666 & $\begin{array}{l}\text { Potent inhibitor of actin polymerization. Inhibits the Arp } 2 / 3 \text {, SpArp2/3 and } \\
\qquad \text { HsArp } / 3 \text { complexes }\end{array}$ & $100.0 \pm 12.9$ & $105.2 \pm 3.0$ \\
\hline & CK869 & $\begin{array}{l}\text { Inhibits actin polymerization vith bovine Arp } 2 / 3 \text { complex and inhibits the } \\
\text { formation of actin filament }\end{array}$ & $17.8 \pm 3.3$ & $34.7 \pm 2.1$ \\
\hline & Latrunculin A & Binds actin monomers preventing incorporation & $36.2 \pm 4.2$ & $51.0 \pm 2.7$ \\
\hline & Latrunculin B & Sequesters $G$-actin and prevent F-actin assembly & $32.0 \pm 6.0$ & $56.7 \pm 2.1$ \\
\hline & Wiskostatin & $N$-WASP inhibitor & $15.7 \pm 6.5$ & $39.8 \pm 15.0$ \\
\hline & $(-)$-Blebbistatin solid & Myosin Il inhibitors, prevents membrane blebbing & $80.4 \pm 9.6$ & $110.9 \pm 3.8$ \\
\hline & CID 1067700 & Inhibitors of nucleotide binding by Ras-related GTPases & $949 \pm 14.6$ & $112.8 \pm 6.4$ \\
\hline & Cucurbitacin E & Inhibits depolymerization of actin filaments & $0.0 \pm 0.0$ & $16.1 \pm 2.3$ \\
\hline & Jasplakinolide & Promotes actin polymerization and stabilizes actin filaments & $20.4 \pm 3.5$ & $24.0 \pm 1.7$ \\
\hline & Phalloidin & $\begin{array}{l}\text { Functions by binding and stabilizing filamentous actin (F-actin) and effectively } \\
\text { prevents the depolymerization of actin fibers }\end{array}$ & $90.5 \pm 4.3$ & $95.5 \pm 6.4$ \\
\hline & $\mathrm{SMIFH} 2$ & $\begin{array}{l}\text { Prevents formin-mediated actin nucleation and processive barbed end } \\
\text { elongation. }\end{array}$ & $76.9 \pm 7.4$ & $100.1 \pm 2.5$ \\
\hline & Swinholide A & Causes actin depolymerization & $1071 \pm 14.5$ & $138.0 \pm 86.2$ \\
\hline & TAS-301 & $\begin{array}{l}\text { Inhibits intimal thickening by blocking Ca2+/calmodulin-dependent protein } \\
\text { kinase II (CaM kinase II) and cytoskeletal depolymerization }\end{array}$ & $107.0 \pm 9.5$ & $103.9 \pm 11.4$ \\
\hline
\end{tabular}

Primary screening of the actin network inhibitors using TZM-bl cells: To identify compounds that have a regulatory effect on HIV-1 infectivity, $6.25 \mu \mathrm{M}$ of each compound was added to $1 \times 10^{5} \mathrm{TZM}$-bl cells cultured in 96-well white plates. After $48 \mathrm{~h}$, the F-Luc activity was measured with a Bright Glo Luciferase Assay kit. Infectivity and cell viability are presented as the mean \pm SD $(n=3)$ compared with no treatment of the inhibitor.

Group I: Compounds that down-regulate the viral infection with low cytotoxicity

Group II: Compounds that up-regulate the viral infection with low cytotoxicity

Group III: Compounds that exhibit severe cytotoxicity and/or no effect on viral infectivity

titration of HIV-1 particles in the supernatant was performed using an HIV-1 p24 ELISA kit (PerkinElmer, Waltham, MA, USA). All the graphical data for the p24 levels are expressed as a relative amount of p24 compared with those obtained in the absence of the compound, and the data are presented as the mean \pm standard deviation (SD) $(n=3)$.

\section{HIV-1 trans-activating protein (Tat) transcriptional assay}

To test the viral transcriptional activity upon the compounds, TZM-bl cells were transiently transfected with $200 \mathrm{ng}$ of Tat expression plasmid (pcDNA-Tat86) at a concentration of $6.25 \mu \mathrm{M}$. At $24 \mathrm{~h}$ after transfection, the Tat-induced firefly luciferase activity was determined by using the Bright-Glo Luciferase Assay System (Promega, Madison, WI, USA) according to the manufacturer's instructions. Statistically analyzed data are expressed as the mean $\pm S D(n=3)$. The data were compared using the Student's $t$ test and $P<0.05$ was considered to indicate significance. All statistical anallyses were performed using Prism software (v. 5.0; GraphPad Software, San Diego, CA, USA). 


\section{RESULTS}

\section{Primary screening of compounds regulating HIV-1 infectivity}

To identify a novel compound capable of regulating HIV-1 infectivity, we screened the inhibitory compounds of the actin cytoskeleton with convenient TZM-bl cells expressing the HIV-1 receptor CD4 and its co-receptor CXCR5/CCR5, and integrating the HIV-1 LTR promoter-driven firefly luciferase gene in the chromosome. The screened compounds included twenty nine inhibitory compounds that target the actin cytoskeleton network, which were loaded into TZM-bl cells at a final concentration of $6.25 \mu \mathrm{M}$ upon HIV-1 infection. After $48 \mathrm{~h}$, F-Luc activity indicating the relative amount of HIV infection, and cytotoxicity induced by the compounds, were measured simultaneously. In this primary blind screen, three compounds were identified that showed a $>50 \%$ inhibition of HIV-1 infection with a $<40 \%$ decrease in cell viability (Table 1). Surprisingly, one of the compounds identified was Chaetoglobosin A, which showed the most potent inhibitory effect without severe cell toxicity under the experimental conditions (16). CK-548 exhibited a moderate inhibitory effect on viral infection without any cytotoxicity, while TR-100 exhibited a potent inhibition of viral infection combined with moderate cell toxicity.

Otherwise, 187-1 N-WASP inhibitor, EHop-016, CID44216842, ML-141, T56-LIMKi and IMM-01 showed increased viral infectivity with a lack of cytotoxicity at a concentration of $6.25 \mu \mathrm{M}$. Interestingly, the cytochalasin family, i.e. cytochalasins (B, $D, E$ and $H$ ), and dihydrocytochalasin B moderately increased viral infectivity with weak cytotoxicity; but cytochalasin A did not exhibit any discernable anti-HIV effect apart from cell toxicity. However, we were unable to find any evidence to suggest that intensively studied compounds with regard to HIV infection, such as latrunculin A, jasplakinolide and wiskostatin, had a specific inhibitory effect on HIV-1 infection without cytotoxicity (Table 1).

\section{Dose-response of compounds in HIV-1 infection}

To confirm the potency of the compounds detected as specific regulators of HIV-1 infection in the primary screening, we further analyzed their dose response using serial dilutions of the compounds with the TZM-bl cell system. Chaetoglobosin A, an inhibitor of the filamentous actin isolated from the marine-derived endophytic fungus Chaetomium globosum (17), was observed to gradually decrease viral infectivity in a dose-dependent manner, with complete inhibition at $25 \mu \mathrm{M}$. Cytotoxicity was mild up to $12.5 \mu \mathrm{M}$, while cell viability dropped suddenly at $25 \mu \mathrm{M}$. The IC 50 (half maximal inhibitory concentration) of Chaetoglobosin A was determined to be approximately $2 \mu \mathrm{M}$. CK-548, which is an inhibitor of the Arp2/3 complex by inserting it into the core of $\operatorname{Arp3}(18)$, also inhibited HIV-1 infection gradually without any cytotoxicity up to $25 \mu \mathrm{M}$, as a IC 50 value of approximately $5 \mu \mathrm{M}$. TR-100, an inhibitor of tropomyosin (integral components of the actin filaments) (19), showed an inhibitory effect on viral infection distinguishable apart from cytotoxicity, but its inhibitory effect on viral infection was insignificant compared to Chaetoglobosin A and CK-548 (Fig. 1A).

On the other hand, eleven compounds exhibiting up-regulation of HIV infection at a single dosage were further tested to define their exact effect on viral infection when using serially diluted concentrations. Among them, EHop-016, a potent and specific inhibitor of Rac1 and Rac3 GTPase activity, increased viral infectivity the most without any cytotoxicity from the lowest concentration to $6.25 \mu \mathrm{M}$, but this increase was suddenly halted with cell death at $12.5 \mu \mathrm{M}$. Both CID44216842 and ML-141 are known as specific inhibitors of Cdc42 GTPase, which also induced viral infectivity in a similar pattern to each other. These enhancements began at concentrations of $3.12 \mu \mathrm{M}$, while cell viability and viral infectivity were almost completely abrogated in both at $25 \mu \mathrm{M}$. Cytochalasins are known to be fungal toxins that bind to the barbed-end of the filamentous actin, and biologically inhibit the assembly and disassembly of monomeric G-actin from the end. With the exception of cytochalasin $A$, most cytochalasins exhibited converse effects on viral infectivity dependent on their concentration. Cytochalasins B, D, E, H and dihydrocytochalasin B showed moderate inhibitory effects on viral infection at below concentrations of $0.78,0.19,0.19,0.78$, and $0.19 \mu \mathrm{M}$ respectively, whereas the infectivity curves were suddenly turned to up-regulation from over these concentrations, these increases of infectivity reached at a maximum of $250 \%$ in the case of cytochalasin D with moderate cytotoxicity. 187, N-WASP inhibitor also significantly induced viral infectivity by up to 
A

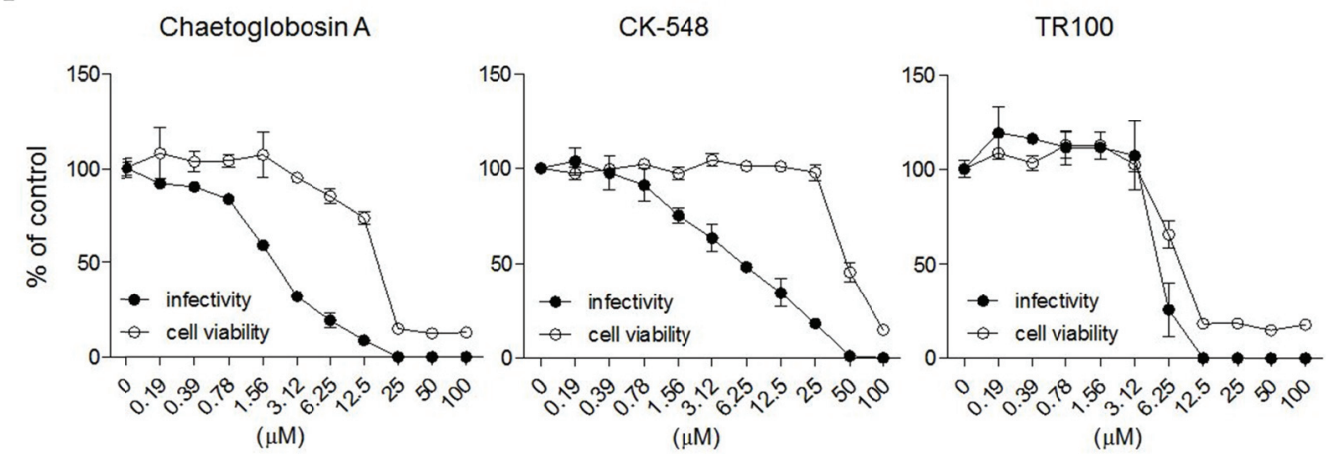

B
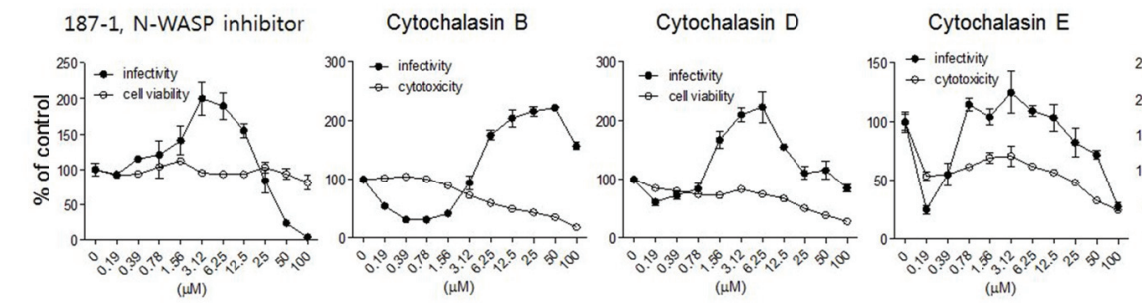

Cytochalasin $\mathrm{H}$

CID44216842

ML 141
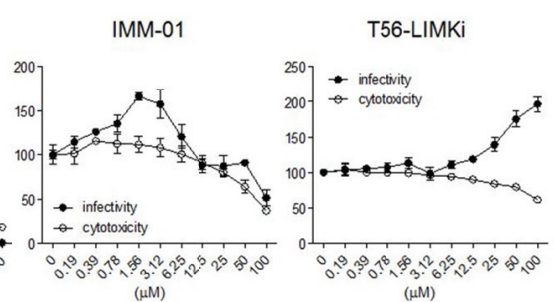

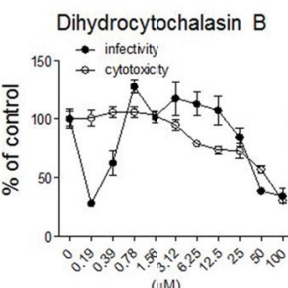

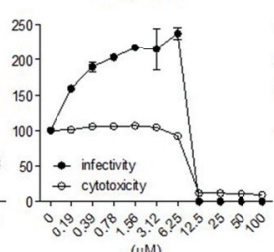

(uM)

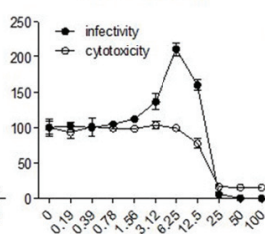

(LM)

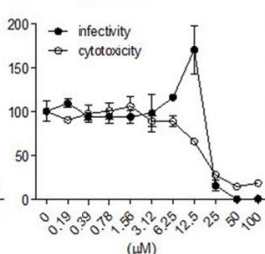

(uM)

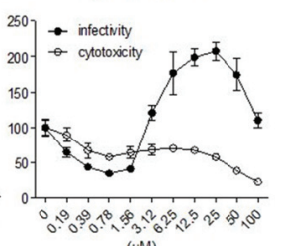

(uM)

Figure 1. Dose-responsive effect on viral infectivity of compounds selected from primary screening $1 \times 10^{4} \mathrm{TZM}$-bl cells were cultured in a $100 \mu \mathrm{L}$ medium for $24 \mathrm{~h}$ and then serial dilutions of the inhibitory (A) and inducible (B) compounds of HIV-1 infection were added to the cells. At $48 \mathrm{~h}$ after treatment, the activity of luciferases was determined as described in the Materials and Methods section.

two times within a concentration range of $3.12-6.25 \mu \mathrm{M}$. IMM-01, an agonist to mammalian diaphanous (mDia) family formins that are Rho-directed effectors inducing F-actin assembly and stabilization of microtubules in cells (20). IMM-01 treatment increased viral infectivity by a maximum of $170 \%$ at $1.56 \mu \mathrm{M}$ (Fig. 1B). T56-LIMKi, a LIMK inhibitor, increased viral infectivity at a high dosage. Our results indicate that the multiple signals of actin-cytoskeleton dynamics are differently integrated to manifold stages of HIV infection.

\section{Effects on viral production of inhibitors of viral infection}

Recently, it has been reported that particle assembly and budding are accompanied by an actin-driven mechanism. Therefore, this study attempted to assess the effect on the viral production of compounds that exhibited a regulatory effect on viral infection, using persistently HIV-1-infected $\mathrm{ACH} 2$ cells that robustly produce viral particles upon administration of the phorbol myristate acetate (PMA)-ionomycin treatment. As shown in Figure 2, the effects on viral production of these small molecules did not correlate with the inhibition of viral infection (Fig. 3). Interestingly, Chaetoglobosin A was observed to completely inhibit HIV-1 production with no cytotoxicity in the cells. However, CK-548 did not inhibit viral production in contrast to its inhibitory effect on viral infection. The inhibition of viral production by TR-100 was combined with severe cytotoxicity (Fig. 2A). These data indicate that Chaetoglososin A-mediated disruption of actin skeleton signaling effectively inhibited both viral infection and viral production. Otherwise, cytochalasins (B, D, E and H), EHop-016 and ML-141 showing increased viral infection inhibited the viral production without significant cytotoxicity. Interestingly, Dihydrocytochalasin only 
A

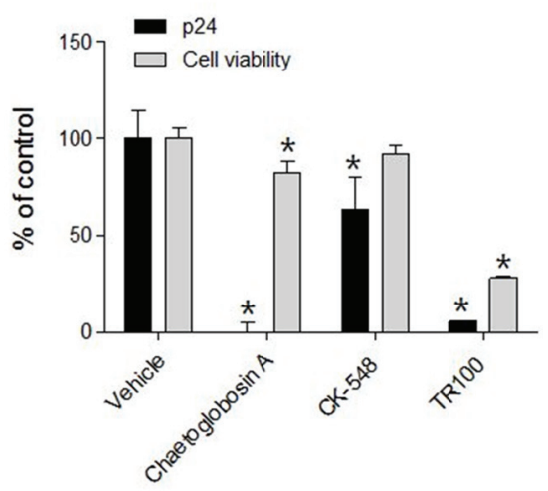

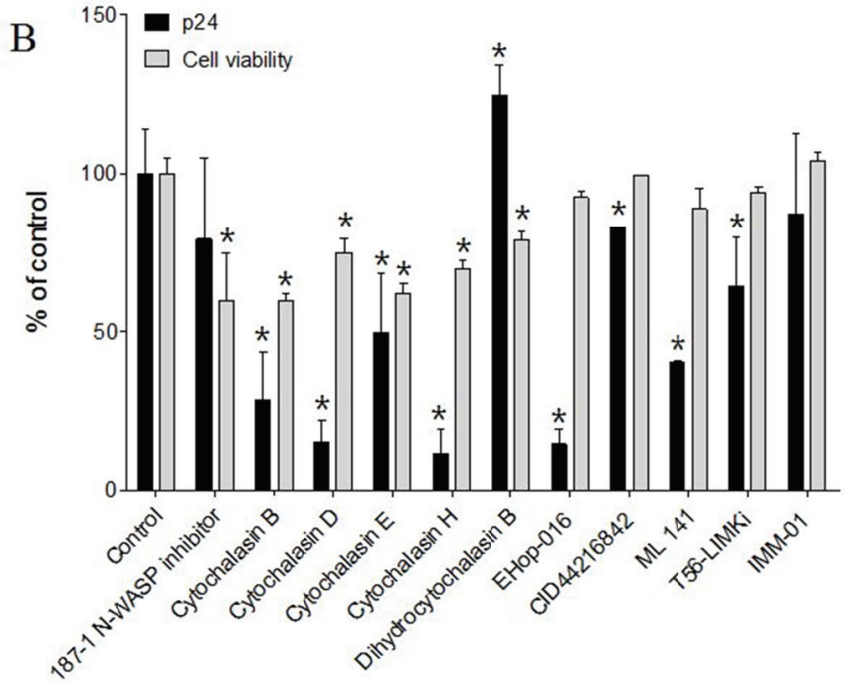

Figure 2. Regulatory effect of compounds on viral productivity. The regulatory effect of inhibitory (A) and inducible (B) compounds of HIV-1 infectivity on viral productivity was assessed with persistently HIV-1-infected cells $\mathrm{ACH} 2.2 \times 10^{4} \mathrm{ACH} 2$ cells were cultured in the presence of compounds at a final concentration of $6.25 \mu \mathrm{M}$ with PMA $(0.5 \mu \mathrm{g} / \mathrm{mL}) /$ ionomycin $(1$ $\mu \mathrm{g} / \mathrm{mL}$ ). At $48 \mathrm{~h}$ after treatment, the titration of HIV-1 particles in the supernatant was performed using an HIV-1 p24 ELISA kit. The data are presented as the mean \pm SD $(n=3) . * P<0.05$ untreated vs. compound -treated cells.

increased viral production (Fig. 2B). These data indicate that actin networks engaged in HIV-1 pathogenesis play different roles according to the circumstances or stage of viral replication.

\section{Effect on Tat-mediated viral transcription by actin dynamic inhibitors}

HIV-1 encodes the Tat, playing a crucial role in viral transcription in its promoter long-term repeat (LTR). The activity of HIV-1 Tat-mediated viral transcription is a key indicator of the viral infectivity assay in this study and is a pivotal step in the expression of viral protein from the integrated provirus following virion assembly and budding in latently HIV-infected cells. Therefore, this study attempted to determine whether these regulators affect the Tat-mediated viral transcription linked to viral infectivity or productivity. TZM-bl cells harboring the viral promoter LTR-driven Firefly-Luciferase were transfected with Tat-expressing plasmids instead of viral infection. As shown in Figure 3A, the Tat-mediated viral transcription was not influenced by Cheatoglobosin A, CK-548, but TR-100 mediating Tat-mediated transcription was slightly decreased with no significance at $6.3 \mu \mathrm{M}$. The compounds, except for the cytochalasin family, showing increase viral infectivity did not showed influence on the Tat-mediated viral transcription. Thus, while cytochalasin B down-regulated it, cytochalasin D and $\mathrm{H}$ slightly induced the Tat-mediated viral transcription. But other compounds showing the enhanced viral infection did not affect the transcription. These data indicate that the inhibitory effects of Chaetoglobosin A and CK-548 on HIV-1 infection and/or production were not linked to viral transcription.

\section{DISCUSSION}

Many antiretroviral drugs that target specific viral proteins, including gp41 fusion, reverse transcriptase, integrase, and viral protease, have been successfully developed (21). However, antiretroviral treatments clearly cannot eliminate the residual latent HIV-1 in resting memory T cells (22). Moreover, is another obvious problem is the emergence of drug-resistant mutations due to the long-term use of these drugs during the lifespan of the patient (23). To overcome these problems, the importance of developing new targets for drugs capable of disrupting HIV replication has been emphasized. In this regard, 
A

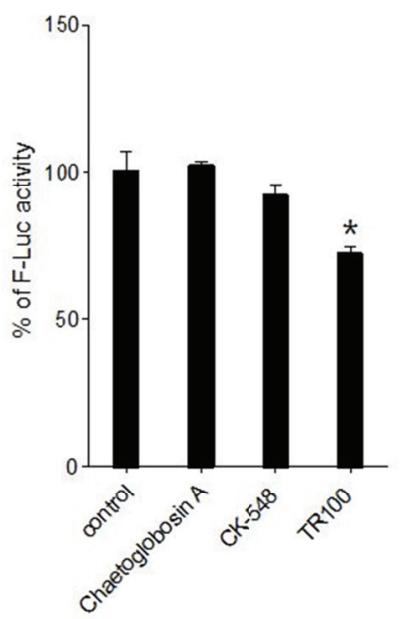

B

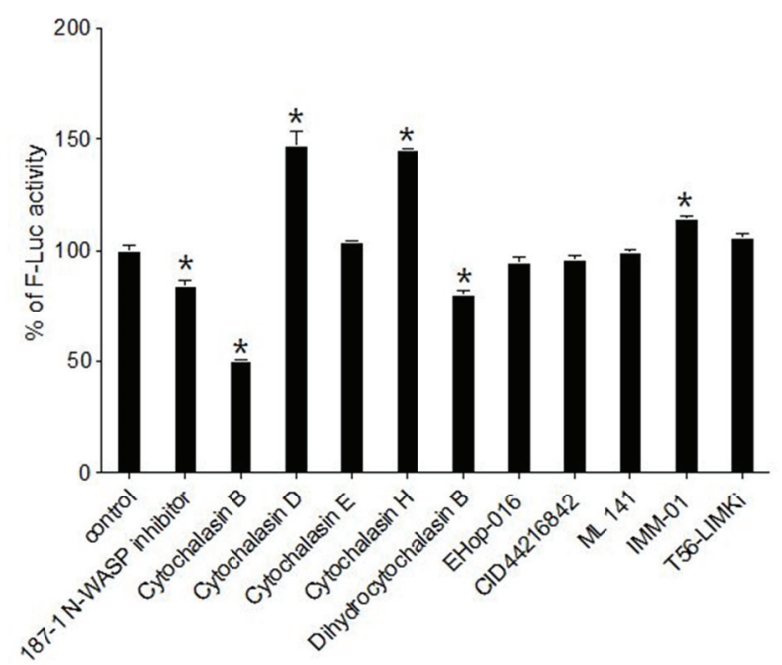

Figure 3. Inhibitory effect on Tat-mediated transcription. The transcriptional regulation of inhilbitory (A) and inducible (B) compounds, as shown in Table 1, was assessed with transient transfection of Tat-expressing plasmid into the TZM-bl cells. 1 $\mu \mathrm{g}$ of Tat-expressing plasmid was introduced into the cells using Lipofectamin 2000 . At $24 \mathrm{~h}$ after transfection, the luciferase activity was measured using a Bright Glo Luciferase Assay kit. The data were represented as relative luciferase activity compared with mock transfection. The data are presented as the mean $\pm \mathrm{SD}(n=3)$. * $P<0.05$ untreated vs. compound treated cells.

parts of the mechanisms underlying actin skeleton dynamics in HIV-1 replication have recently been discovered. Although it is almost certain that actin activity may be important for viral entry processes, including CD4 and CXCR4/CCR5 receptor dynamics, the stabilization of the fusion complex, the promotion of capsid de-coating, and the efficient reverse transcription and migration of PIC require activities of Rac GTPase, PAK, LIMK and cofilin, WAVE2, WASP, Arp2/3, actin etc. in HIV-infected CD4+T cells $(8,9,24,25)$. These studies, hypothesized with scattered findings, were too complicated to understand the exact roles of the pathway of actin dynamics in HIV replication. In addition, some of the findings were controversial because the study used limited inhibitory compounds which might be accompanied by an unexpected disruption of certain signal pathways. Therefore, the results could not be used to elucidate the precise mechanism of HIV-1 infection or a potent actin pathway for HIV infection treatment.

In the present study, small molecules that inhibit the actin cytoskeleton were screened to improve understanding of the interaction mechanism between HIV-1 and the actin dynamic pathway, and to provide new information for developing a potent inhibitor against HIV-1 infection utilizing the actin pathway. Three inhibitory compounds against HIV-1 infection were identified from among twenty-nine inhibitors of the actin skeleton network. Of these, CK-548, an Arp2/3 inhibitor, was previously described as having an inhibitory effect on HIV-1 infection. CK-548 was observed to have a specific inhibitory effect on viral infection without cytotoxicity, but did not show an inhibitory effect on viral production (Figs. 1 and 2). It has been reported that the knock-down of Arp3 results in a dramatic inhibition of HIV-1 replication and that the CK-548 treatment inhibits the Arp2/3-mediated nuclear migration of the HIV pre-integration complex, followed by a blocking of HIV infection without cytotoxicity (25). Consistently with our data, these data demonstrated that the actin filament branching of Arp2/3 may help inhibit HIV infection. Chaetoglobosin A and TR-100 were newly identified as inhibitors having an anti-HIV effect. Chaetoglobosin A, a fungal toxin (26), exhibited the most potent inhibition of HIV-1 infection and production with no cytotoxicity. It is also known to target the filamentous actin, and exhibits both antibacterial and anti-cancer activity. In our data, the compound exhibited weak cytotoxicity up to $12.5 \mu \mathrm{M}$, showing complete inhibition of viral infection and production. This result may explain why the actin network signal targeted by Chaetoglobosin $A$ is specifically necessary to enable viral infection and production. However, other inhibitors targeting the actin filament, including cucurbitacin $\mathrm{E}$ and 
jasplakinolide, showed severe cytotoxicity despite their inhibitory effects on HIV-1 infection, while phalloidin did not have any effect on either HIV-1 infection or cytotoxicity (Table 1). These data may suggest the existence of undefined molecular mechanisms that target the filamentous actin between the inhibitors. TR-100, a factor dissociating actin from tropomyosin-directed actin dynamics (19), also showed a complete inhibitory effect on viral infection at $12.5 \mu \mathrm{M}$, but HIV-1 inhibition was combined with $80 \%$ cell death. Although TR-100's inhibitory effect on HIV infection was not significantly distinguished from cytotoxicity. It seems fairly certain that tropomyosin-related actin dynamics might be partially involved in HIV-1 infection.

In this study, the screening also included two small, well-defined molecule inhibitors that negatively regulate HIV-1 infection, i.e. jasplakinolide, a stabilizer of the filamentous actin obtained from sponge, and latrunculin $A$, which induces de-polymerization by binding to $G$ actin. These compounds have been extensively used in an effort to probe their anti-HIV effects. Some studies showed that jasplakinolide and latrunculin A inhibited HIV-1 infection at $129 \mathrm{nM}$ (and blow) and at 2.5 $\mu \mathrm{M}$, respectively $(10,27)$. However, the data did not accurately define the degree of cytotoxicity at the given concentrations. In the present study's results, although the compounds exhibited an inhibitory effect on HIV-1 infection at similar dosages to previous studies, their effects were accompanied by a parallel level of cytotoxicity in the dose response assay (Table 1; data not shown). One study suggested that wiskostatin, a N-WASP inhibitor, inhibited HIV-1 entry into the macrophage (12); however our results showed a high level of cytotoxicity combined with inhibition of viral infection. Also, another report demonstrated that certain inhibitors caused down-regulation of the ATP level non-selectively and might have had global effects on the cells (28). As such, the exact inhibitory effect on viral infection by wiskostatin could not be defined in this study. In addition, in this study another peptide inhibitor of N-WASP, 187-1, was observed to exhibit enhanced viral inactivity with no cytotoxicity. It has been reported that HIV-1 triggers the nucleation-promoting factor (NPF) WAVE2 Phosphorylation Mediating Arp2/3-dependent PIC migration after entry (25). However, the HIV-1 regulatory role of N-WASP, another well-known NPF, has not been elucidated. The results of this study indicate that N-WASP might inhibit viral infection in contrast to WAVE2. Cytochalasins have also been widely used as inhibitors to probe the actin pathway utilization of HIV-1 (29, 30). Several studies reported that 5 uM of cytochalasin D dramatically inhibited viral DNA synthesis (11) and that cytochalasin $D$ and cytochalasin B inhibited viral fusion $(31,32)$. However, no inhibitory effect of viral infection by cytochalasin D, B was observed at the concentrations used by those studies in this study. Otherwise, most cytochalasins, including cytochalasin $D$ and B, showed enhanced infectivity by as much as 2.5 times at over $3 \mu \mathrm{M}$, except cytochalasin A. As an inhibitory pattern of viral infection was only observed at low concentrations of these compounds, it is possible that this was caused by the inhibition of viral fusogenic activity similar to their anti-HIV activity as reported in previous studies. It should be noted here that the enhanced viral infectivity caused by a higher concentration of these compounds may reveal that the actin network is more dynamically regulated by HIV than previously thought. Furthermore, the results of this study showed that the Rho GTPasse family, including EHop-016, CID44216842 and ML-141, greatly enhanced HIV-1 infectivity with a similar pattern in each. The Rac GTPase inhibitor EHop-016 exhibited increased viral infectivity from a low concentration, whereas the cdc42 inhibitors only showed a great increase of viral infection within a range of $6.25 \mu \mathrm{M}-12.5 \mu \mathrm{M}$. Although numerous studies have attempted to clarify the role of actin dynamics in HIV-1 infection, the roles of the Rho GTPase family, which act upstream actin dynamics, has been poorly elucidated with regard to HIV infection. Our data demonstrate that the Rho GTPase family may play critical roles in HIV-1 infection and, furthermore, that the controlled signal from the GTPase family may inhibit the process of viral infection. An increase in viral infection was also observed following treatment with a high concentration of T56-LIMK1, an inhibitor for LIMK, which transports Rho GTPase signals to downstream effectors, the combined our data may suggest that signals from Rho GTPase negatively regulate HIV infection. Conversely, one study attributed the positive role of LIMK in HIV-1 infection to LIMK activation with okadaic acid, which led to a dramatic increase of HIV infection in CD4+ T cells (24). However, it is thought that the pan kinase activator used in this study might have raised the global cell activation linked to improved HIV infection with non-specific.

As the process of HIV-1 infection involves many complex signal cascades with a variety of host cellular factors, defining the exact roles of the actin network in HIV-1 infection was a complex issue. Additionally, the results from previous studies sometimes conflicted with or contradicted the results of this study, but it may be the case that some inhibitors of actin 
dynamics used in previous studies exhibited non-specific inhibition targeting other host factors instead of the known target. Therefore, this study elucidated the exact effects of actin dynamics inhibitors on HIV-1 infection.

In conclusion, the present study has precisely identified several inhibitors of actin dynamics that have a regulatory effect on HIV-1 infection with no cytotoxicity. It has also confirmed the inhibitory effects of CK-548, and identified the new inhibitory roles of Chaetoblobosin A and TR-100 in HIV-1 infection and production. Furthermore, this study demonstrates that most cytochalasins act inversely on HIV infection according to their concentrations, and that some inhibitors of Rho GTPase; EHop-016, CID44216842 and ML-141, and LIMK significantly increase viral infection within a range of several $\mu \mathrm{M}$. Taken together, the results of this study suggest that the newly identified roles of these inhibitors of HIV-1 infection may be helpful in understanding complicated actin dynamics during HIV-1 infection and provide important information for the development of potent new anti-HIV drugs targeting the actin network for HIV infection.

\section{Acknowledgments}

We are grateful to the AIDS Research and Reference Reagent Program (ARRRP, NIH, USA) for providing the cell lines (TZM-bl, A3.01, ACH-2, Jurkat and J1.1 cells) and the HIV-1NL4-3 strain. This work was supported by grants from the Korea National Institute of Health (Grant Number: 2017-NI51001-00).

\section{REFERENCES}

1) Gallo RC, Salahuddin SZ, Popovic M, Shearer GM, Kaplan M, Haynes BF, et al. Frequent detection and isolation of cytopathic retroviruses (HTLV-III) from patients with AIDS and at risk for AIDS. Science 1984;224:500-3.

2) World Health Organization USCfDCaP, The Global Fund to Fight AIDS, Tuberculosis and Malaria. HIV drug resistance report 2017. World Health Organization 2017.

3) Lee SH, Dominguez R. Regulation of actin cytoskeleton dynamics in cells. Mol Cells 2010;29:311-25.

4) Blanchoin L, Boujemaa-Paterski R, Sykes C, Plastino J. Actin dynamics, architecture, and mechanics in cell motility. Physiol Rev 2014;94:235-63.

5) Frischknecht F, Moreau V, Röttger S, Gonfloni S, Reckmann I, Superti-Furga G, et al. Actin-based motility of vaccinia virus mimics receptor tyrosine kinase signalling. Nature 1999;401:926-9.

6) Goley ED, Ohkawa T, Mancuso J, Woodruff JB, D'Alessio JA, Cande WZ, et al. Dynamic nuclear actin assembly by Arp2/3 complex and a baculovirus WASP-like protein. Science 2006:314:464-7.

7) Stolp B, Reichman-Fried M, Abraham L, Pan X, Giese SI, Hannemann S, et al. HIV-1 Nef interferes with host cell motility by deregulation of Cofilin. Cell Host Microbe 2009;6:174-86.

8) Spear M, Guo J, Wu Y. The trinity of the cortical actin in the initiation of HIV-1 infection. Retrovirology 2012;9:45.

9) Spear M, Guo J, Wu Y. Novel anti-HIV therapeutics targeting chemokine receptors and actin regulatory pathways. Immunol Rev 2013:256:300-12.

10) Yoder A, Yu D, Dong L, lyer SR, Xu X, Kelly J, et al. HIV envelope-CXCR4 signaling activates cofilin to overcome cortical 
actin restriction in resting CD4 T cells. Cell 2008;134:782-92.

11) Bukrinskaya A, Brichacek B, Mann A, Stevenson M. Establishment of a functional human immunodeficiency virus type 1 (HIV-1) reverse transcription complex involves the cytoskeleton. J Exp Med 1998;188:2113-25.

12) Carter GC, Bernstone L, Baskaran D, James W. HIV-1 infects macrophages by exploiting an endocytic route dependent on dynamin, Rac1 and Pak1. Virology 2011:409:234-50.

13) Haller C, Rauch S, Michel N, Hannemann S, Lehmann MJ, Keppler OT, et al. The HIV-1 pathogenicity factor Nef interferes with maturation of stimulatory T-lymphocyte contacts by modulation of N-Wasp activity. J Biol Chem 2006:281:19618-30.

14) Kim HY, Choi BS, Kim SS, Roh TY, Park J, Yoon CH. NUCKS1, a novel Tat coactivator, plays a crucial role in HIV-1 replication by increasing Tat-mediated viral transcription on the HIV-1 LTR promoter. Retrovirology 2014;11:67.

15) Shin $Y$, Yoon $\mathrm{CH}$, Yang HJ, Lim H, Choi BS, Kim SS, et al. Functional characteristics of the natural polymorphisms of HIV-1 gp41 in HIV-1 isolates from enfuvirtide-naive Korean patients. Arch Virol 2016;161:1547-57.

16) Shin Y, Choi BS, Kim KC, Kang C, Kim K, Yoon CH. Development of a dual reporter screening assay for distinguishing the inhibition of HIV Tat-mediated transcription from off-target effects. J Virol Methods 2017:249:1-9.

17) Kawahara $T$, Itoh $M$, Izumikawa $M$, Sakata $N$, Tsuchida $T$, Shin-ya K. New chaetoglobosin derivatives, MBJ-0038, MBJ-0039 and MBJ-0040, isolated from the fungus Chaetomium sp. f24230. J Antibiot (Tokyo) 2013;66:727-30.

18) Nolen BJ, Tomasevic N, Russell A, Pierce DW, Jia Z, McCormick CD, et al. Characterization of two classes of small molecule inhibitors of Arp2/3 complex. Nature 2009;460:1031-4.

19) Bonello TT, Janco M, Hook J, Byun A, Appaduray M, Dedova I, et al. A small molecule inhibitor of tropomyosin dissociates actin binding from tropomyosin-directed regulation of actin dynamics. Sci Rep 2016:6:19816.

20) Arden JD, Lavik KI, Rubinic KA, Chiaia N, Khuder SA, Howard MJ, et al. Small-molecule agonists of mammalian Diaphanous-related (mDia) formins reveal an effective glioblastoma anti-invasion strategy. Mol Biol Cell 2015;26: 3704-18.

21) De Clercq E, Li G. Approved Antiviral Drugs over the Past 50 Years. Clin Microbiol Rev 2016:29:695-747.

22) Churchill MJ, Deeks SG, Margolis DM, Siliciano RF, Swanstrom R. HIV reservoirs: what, where and how to target them. Nat Rev Microbiol 2016;14:55-60.

23) Margolis AM, Heverling $H$, Pham PA, Stolbach A. A review of the toxicity of HIV medications. J Med Toxicol 2014;10:26-39.

24) Vorster PJ, Guo J, Yoder A, Wang W, Zheng Y, Xu X, et al. LIM kinase 1 modulates cortical actin and CXCR4 cycling and is activated by HIV-1 to initiate viral infection. J Biol Chem 2011:286:12554-64.

25) Spear M, Guo J, Turner A, Yu D, Wang W, Meltzer B, et al. HIV-1 triggers WAVE2 phosphorylation in primary CD4 T cells and macrophages, mediating Arp2/3-dependent nuclear migration. J Biol Chem 2014;289:6949-59.

26) Sekita S, Yoshihira K, Natori S, Kuwano H. Structures of chaetoglobosin A and B, cytotoxic metabolites of chaetomium 
globosum. Tetrahedron Letters 1973;14:2109-12.

27) Wang W, Guo J, Yu D, Vorster PJ, Chen W, Wu Y. A dichotomy in cortical actin and chemotactic actin activity between human memory and naive $T$ cells contributes to their differential susceptibility to HIV-1 infection. J Biol Chem 2012:287:35455-69.

28) Guerriero CJ, Weisz OA. N-WASP inhibitor wiskostatin nonselectively perturbs membrane transport by decreasing cellular ATP levels. Am J Physiol Cell Physiol 2007;292:C1562-6.

29) Jernigan KM, Blumenthal R, Puri A. Varying effects of temperature, $\mathrm{Ca}(2+)$ and cytochalasin on fusion activity mediated by human immunodeficiency virus type 1 and type 2 glycoproteins. FEBS Lett 2000;474:246-51.

30) Kondo N, Marin M, Kim JH, Desai TM, Melikyan GB. Distinct requirements for HIV-cell fusion and HIV-mediated cell-cell fusion. J Biol Chem 2015;290:6558-73.

31) Frey S, Marsh M, Günther S, Pelchen-Matthews A, Stephens P, Ortlepp S, et al. Temperature dependence of cell-cell fusion induced by the envelope glycoprotein of human immunodeficiency virus type 1. J Virol 1995;69:1462-72.

32) lyengar S, Hildreth JE, Schwartz DH. Actin-dependent receptor colocalization required for human immunodeficiency virus entry into host cells. J Virol 1998;72:5251-5. 